\title{
Inhalt, Vol. 6, No. 1, 1983
}

\section{Hinweise für Autoren/Sonderbände 2}

Impressum 3

Bernsmeier, R.; Holzgrebe, E.; Luck, P.; Bruhn, H. D. und Zurborn, K. H., Kiel

Thrombin, Faktor XIII und Fibronectin als Regulatoren der Proliferation von Tumorzellen und Gefäßwandzellen 4

Dimitriadis, K.; Boutis, L.; Tavantzis, J.;

Mouratidou, D.; Bouchoris, N.; Koukourikos, S.;

Hatzigogos, K. und Safariní, S.,

Thessalonikii Griechenland

Chemotherapie des nicht-kleinzelligen Bronchialkarzi-

noms 8

Blum, U.; Ungeheuer, E. und Wacha, H., Frankfurt/M.

Bedeutung der klinischen Symptomatik für die Früh-

erkennung des Bronchialkarzinoms 12

Schütte, J.; Bremer, K.; Niederle, N.; Schoetensack, B.; Schmidt, C. G. und Seeber, S., Essen

Sequentiell-alternierende Chemotherapie nicht-semino-matöser Hodentumoren mit

Adriamycin/Cisplatin und Bleomycin/Vinblastin - Therapieansprechen und -versa-gen in

Abhängigkeit von Histologie und Tumorstadium -. 16

Steinke, B.; Voss, A. C; Ostendorf, P. und

Waller, H. D., Tubingen

Kombinierte Chemo- und Radiotherapie des kleinzelli-

gen Bronchialkarzinoms mit Anwendung hoher Strahlen-

dosen 22

Winkler, K.; Beron, G.; Thöne, I.; Müller-Wickup, J. und Landbeck, G., Hamburg, Jürgens, H. und Goebel, U., Düsseldorf, Gutjahr, P., Mainz, Spaar, H.-J., Bremen, Drescher, J., Oldenburg und Thomas, P., Krefeld

Therapie der akuten Lymphoblastenleukämie im Kindes-

alter - Multizentrische prospektive Therapiestudie

COALL-80 26

Neuigkeiten für die klinische Praxis 34

Gericke, D., Frankfurt, Hofstetter, A.; Keiditsch, E. und Lersch, Chr., München

Möglichkeiten immunologischer Beeinflussung des

Brown-Pearce-Karzinoms am Kaninchen 36

Buchbesprechungen 44

Onkologie-Mitteilungen $\quad 45$

Contents

Instructions for Authors/Special Editions 2

Imprint 3

Bernsmeier, R.; Holzgrebe, E.; Luck, P.; Bruhn, H. D., and Zurborn, K. H., Kiel 
Growth Regulation of Tumor Cells and of Vessel Wall

Cells by Thrombin, Factor XIII and Fibronectin 4

Dimitriadis, K.; Boutis, L.; Tavantzis, J.; Mouratidou, D.; Bouchoris, N.; Koukourikos, S.;

Hatzigogos, K. , and Safarini, S. , Thessalonikii Greece

Chemotherapy of Non-Small Cell Bronchogenic Carci

noma 8

Blum, U.; Ungeheuer, E., and Wacha, H., Frankfurt/M.

Worth of Clinical Symptoms for Early Detection of Lung

Cancer 12

Schütte, J.; Bremer, K.; Niederle, N.; Schoetensack, B.;

Schmidt, C. G., and Seeber, S., Essen

Alternating Sequential Combination Chemotherapy in

Malignant Testicular Teratomas with Adriamycin/Cis

platin and Bleomycin/Vinblastine 16

Steinke, B.; Voss, A. C; Ostendorf, P., and

Waller, H. D., Tubingen

Combination Therapy of Small Cell Lung Cancer under

High Dose Radiation 22

Winkler, K.; Beron, G.; Thöne, I.; Müller-Wickup, J., and Landbeck, G., Hamburg, Jürgens, H., and

Goebel, U., Düsseldorf, Gutjahr, P., Mainz,

Spaar, H.-J., Bremen, Drescher, J., Oldenburg, and

Thomas, P., Krefeld

Therapy of Childhood Acute Lymphoblastic Leukemia -

Multicenter Prospective Trial COALL-80 26

News for Clinical Practice 34

Gericke, D., Frankfurt, Hofstetter, A.; Keiditsch, E., and

Lersch, Chr., München

Possibilities of Immunotherapy or Immune Prophylaxis

of the Brown-Pearce Carcinoma in Rabbits 36

Book Reviews 44

Oncology Communications 45

Beilagen-Hinweis:

Diese Ausgabe enthält eine Beilage der PINO Pharmazeutische Präparate GmbH. Wir bitten um gfl. Beachtung.

Bibliographischer Hinweis: Inhaltsverzeichmsse dieser Zeitschrift erscheinen regelmäßig in current contents ${ }^{\circledR}$ sowie in anderen bibliographischen Diensten. 